\title{
Early Primary Open-Angle Glaucoma
}

\author{
Austin R. Lifferth, OD, FAAO \\ Veterans Affairs, \\ Outpatient Clinic, \\ The Villages, FL, USA
}

\section{Introduction}

Glaucoma is the leading cause of irreversible blindness worldwide and is projected to affect more than 79.6 million people by 2020 , over $10 \%$ of whom will be bilaterally blind. ${ }^{1}$

This multifactorial progressive optic neuropathy causes characteristic retinal nerve fiber layer damage that will eventually lead to associated glaucomatous visual field defects if left untreated. Unfortunately, these visual field defects are difficult for the patient to detect until more advanced stages and, as a result, early glaucoma is usually asymptomatic. ${ }^{2}$

This paper presents a case that is consistent with population studies that suggest that as many as half of people with glaucoma are unaware that they have the disease. ${ }^{3}$

\section{CASE REVIEW}

A healthy 71-year-old Caucasian male reported to our office as a new patient with complaints of mild blurry vision, $\mathrm{OD}=\mathrm{OS}$, at both distance and near.

The patient reported that he had had an eye exam "6-7 years" prior to our exam and was told that he had "symptoms of glaucoma"; however, he was not diagnosed with glaucoma and did not receive additional treatment. He denied any family history of glaucoma, was in good general health, and reported no other difficulties involving either eye.

The patient was self-medicating with 81mg aspirin, multi-vitamins, fish oil capsules, vitamin B complex, and red yeast rice capsules, all taken once daily.

His most recent blood pressure was 150/67 at 14:05 in the seated position, while his A1C was 5.8\% and his blood glucose level was $116 \mathrm{mg} / \mathrm{dL}$ at 14:37. His body mass index was 28 . No allergies to any medications were reported.

The patient's unaided entering distance visual acuities and pinhole acu-

ities $(\mathrm{PH})$ were:

OD: $20 / 60-1$ PH: $20 / 25$

OS: 20/40+2 PH: 20/25+2

Subjective refraction and best-corrected visual acuities were:

OD: $-0.75-1.50 \times 07020 / 20$

OS: $-0.25-1.50 \times 06220 / 25+1$

Preliminary testing showed that the pupils were equally round and reactive to light with no relative afferent pupillary defect. Confrontation visual fields were full to finger count OD, OS. Motility testing was full without restriction or pain OD, OS. 
Slit lamp examination showed mild bilateral blepharitis, clear conjunctiva, and clear cornea without endothelial pigment or keratic precipitates. The anterior chamber was deep and quiet by Van Herick angle estimation and the iris was normal without signs of atrophy, obvious posterior synechiae, or trans-illumination defects. Intraocular pressures (IOP) were 17 OD, 15 OS at 08:01 by Goldmann applanation tonometry.

Dilated examination showed trace nuclear sclerosis and cortical opacities without evidence of pseudoexfoliation or pigment. The macula, vessels, and periphery were all normal OD, OS. There was a posterior vitreous detachment OD, OS with no evidence of peripheral retinal abnormality.

The optic nerve head was average to large size OD>OS based on the vertical disc height using the adjusted slit lamp graticule and a Volk $78 \mathrm{D}$ lens with a correction factor of 1.2x. The optic cup was of moderate depth, with early visible laminar dots OU. There was mild alpha zone parapapillary atrophy, but no signs of pallor or disc hemorrhages OU. There was a subtle inferior retinal nerve fiber layer wedge defect with associated inferior rim thinning, inferior vessel baring, and inferior arteriole narrowing OU. Additionally, the superior rim was suspicious for glaucomatous optic neuropathy OU with evidence of early vessel baring OD $>$ OS and relative thinning compared to other optic nerve sectors. Cup-to-disc ratios were estimated to be $0.7 \mathrm{v} / 0.7 \mathrm{~h}$ OD and $0.75 \mathrm{v} / 0.7 \mathrm{~h}$ OS.

Baseline photos and optical coherence tomography (OCT) Optic Nerve Head (ONH) and Retinal Nerve Fiber Layer (RNFL) Analysis were acquired. Both subjective and objective imaging confirmed the findings in the clinical exam, as shown in Figures 1 and 2.

Figure 1: Retinal images showing inferior-temporal localized retinal nerve fiber layer defects with associated inferior-temporal neuroretinal rim thinning. Note the early relative superior neuroretinal rim thinning $O U$.

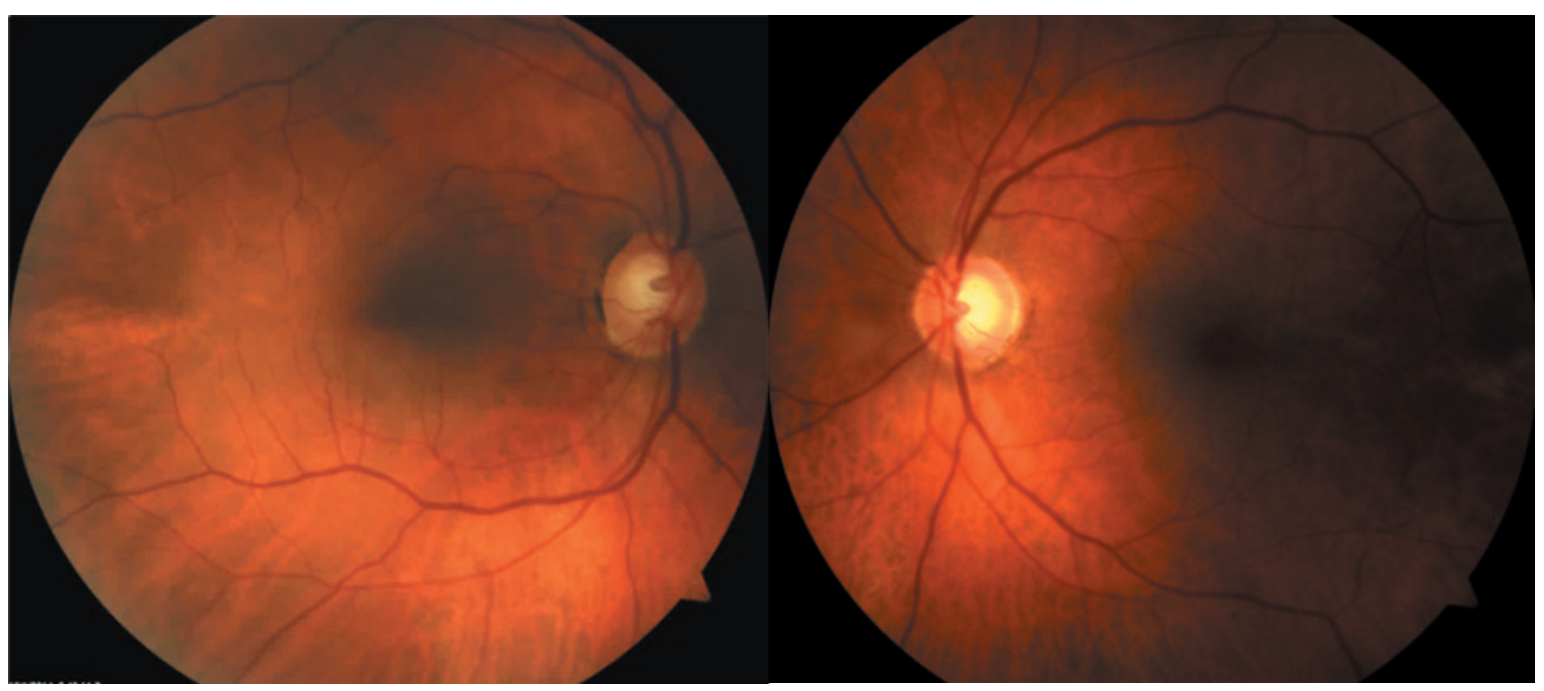

The patient was given a provisional diagnosis of early glaucoma, OS $>\mathrm{OD}$, and asked to return within 1 month for repeat IOP measurements with baseline gonioscopy, pachymetry, and threshold visual field testing.

At the one-month follow-up appointment, the patient was found to have stable acuities without any additional ocular complaints. His intraocular pressures were slightly higher than baseline, at 21 OD, 19 OS at 10:42 am by Goldmann applanation tonometry. Pachymetry yielded central corneal thickness measurements that were slightly thinner than average, at $524 \mathrm{u}$ OD, $525 \mathrm{u}$ OS. Gonioscopy showed that the ciliary body was visible in all four quadrants with flat iris insertion, light trabecular meshwork pigmentation, and no evidence of peripheral anterior synechiae or angle recession OD, OS. 
Figure 2: Optical coherence tomography (OCT) Optic Nerve Head (ONH) and Retinal Nerve Fiber Layer (RNFL) Analysis $O U$. Note the bilateral nasal posterior vitreous detachments with associated artifacts observed on the RNFL Thickness and Deviation Maps. Furthermore, the RNFL thinning is localized and, as such, is only noted on the RNFL Clock Hours Map while appearing as "normal" on the RNFL Quadrant Map.

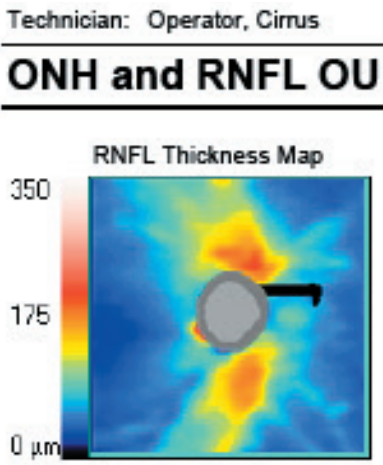

RNFL Deviation Map

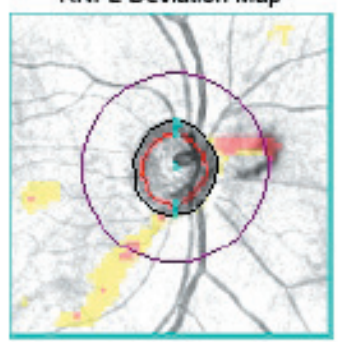

Disc Center $(0.06,0.15) \mathrm{mm}$ Extracted Horizontal Tomogram

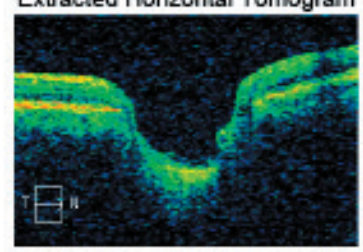

Extracted Vertical Tomogram

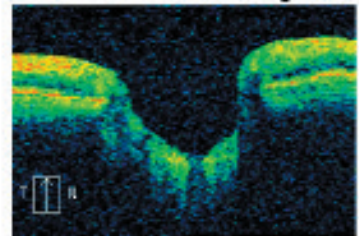

RNFL Circular Tomogram

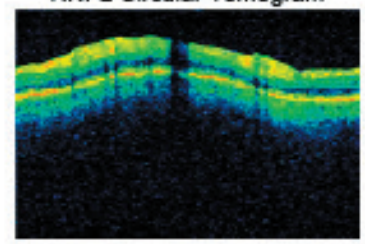

Signal Strength: $8 / 10$

$8 / 10$

\begin{tabular}{|c|c|c|}
\hline$\Delta$ & OD & OS \\
\hline Axerage RNFL Thizkness & $87 \mu \pi$ & $86 \mu \mathrm{n}$ \\
\hline FNFL Symetry & \multicolumn{2}{|c|}{$90 \%$} \\
\hline Rin Ares & $0.06 \mathrm{~mm}^{2}$ & $0.05 \mathrm{~mm}$ \\
\hline Disc Area & $2.14 \mathrm{~mm}^{2}$ & $1.88 \mathrm{~mm}^{2}$ \\
\hline Average C.D Rato & 0.74 & 0.71 \\
\hline Vetica C.D Ratio & 0.78 & 0.78 \\
\hline Cup voute & $0.588 \mathrm{~mm}^{2}$ & $0.412 \mathrm{~mm}$ \\
\hline
\end{tabular}

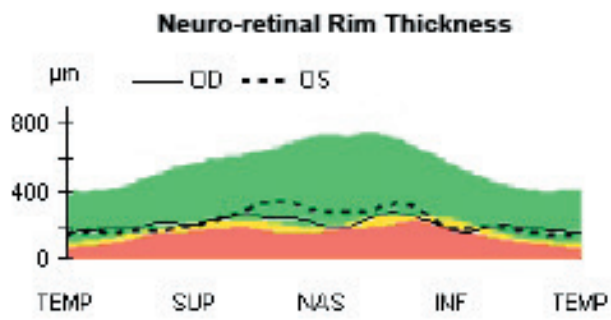

RNFL Thickness
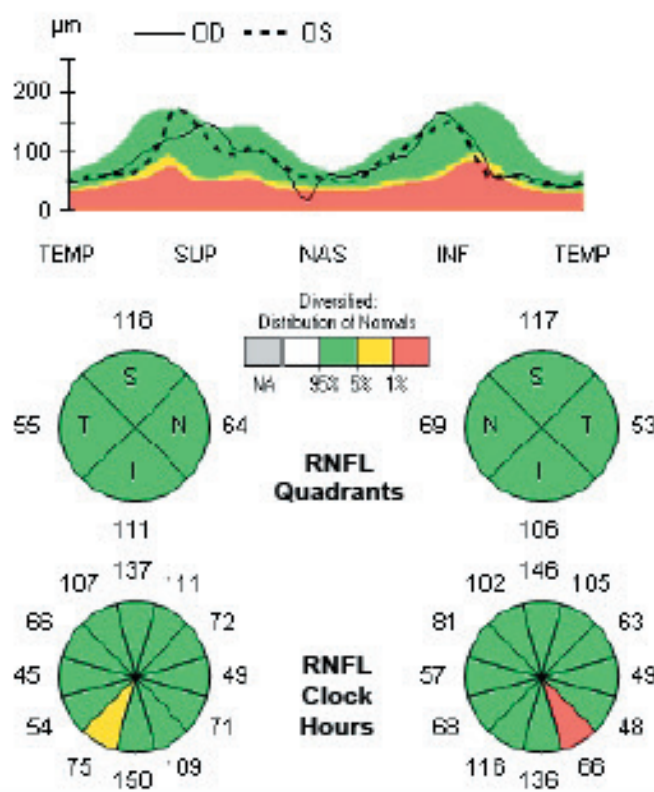

RNFL

Clock

Hours

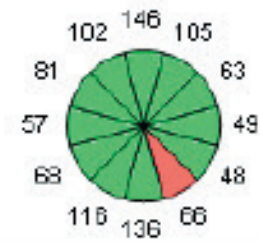

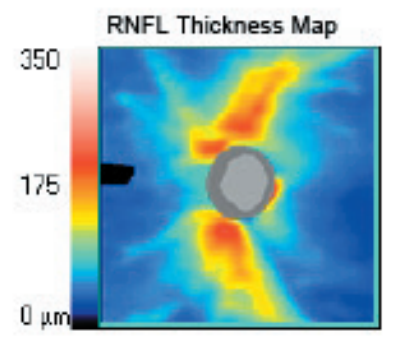

RNFL Deviation Map

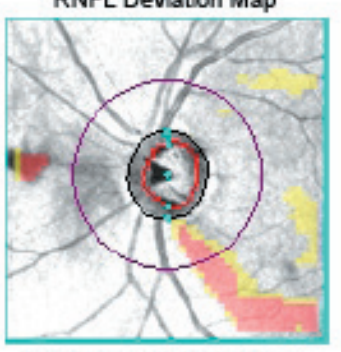

Disc Center(0.03,0.12)mm Extracted Horizontal Tomogram

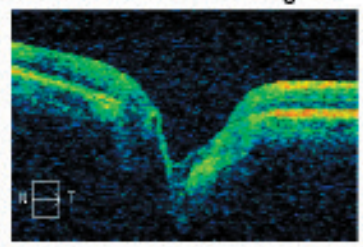

Extracted Vertical Tomogram

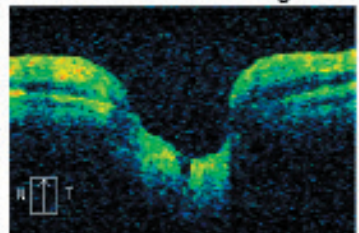

RNFL Circular Tomogram

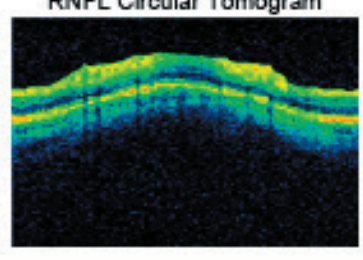


Baseline threshold visual field testing (Humphrey 24-2 SITA Fast) showed normal-sensitivity OD with good reliability ( $0 / 10$ fixation losses, $5 \%$ false positives, and $0 \%$ false negatives) and several focally depressed superior nasal defects OS with lower reliability (5/11 fixation losses, $3 \%$ false positives, and $8 \%$ false negatives).

Figure 3: Baseline Humphrey 24-2 SITA Fast visual field plots show focally depressed defects OS only.
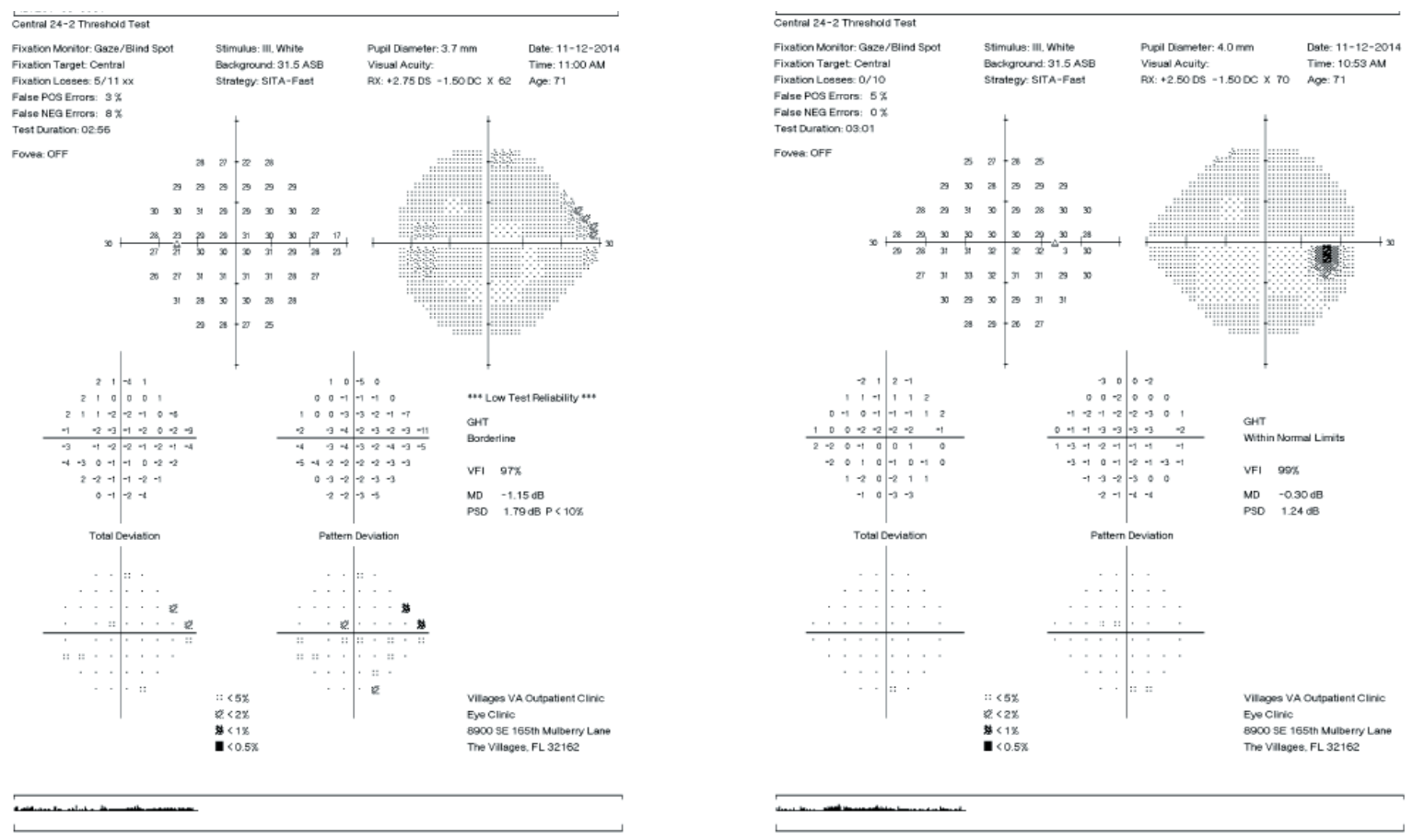

Due to the normal gonioscopic appearance of the angle, the patient was given a more specific diagnosis of early primary open angle glaucoma OS $>$ OD with questionable early perimetric defects OS based on the SITA Fast testing algorithm. Bengtsson and Heijl ${ }^{4}$ found that SITA Fast test times (avg 5.0 min) were significantly shorter than Full Threshold (avg $14.6 \mathrm{~min}$ ) and Fastpac (avg $9.4 \mathrm{~min}$ ) test times, but were essentially equal in terms of reproducibility. However, as expected, the sensitivity for detecting shallow (subtle) defects was greater with Full Threshold testing. In other words, shallow defects noted in Full Threshold testing were progressively less visible (perhaps even absent) with Fastpac and SITA Fast, while the detection of deeper focal defects was essentially identical with all three strategies.

Through collaboration with the patient, we decided to monitor the condition closely at that time without treatment until repeat structural and functional testing confirmed the suspected defects (and/or suggested progression) and to further establish baseline intraocular pressures at various diurnal time points. However, based on the case findings thus far, and based on the Early Manifest Glaucoma Trial (EMGT) and the Collaborative Normal Tension Glaucoma Study (CNTGS), treatment will likely be initiated to help reduce the risk of disease progression. Furthermore, and perhaps more specific to our patient, Kim et al. found that sufficient IOP reduction slowed disease progression even in patients with suspected preperimetric glaucoma. ${ }^{5}$

The patient was asked to return to our office in 3-4 months for repeat tonometry, pachymetry, and 24-2 threshold visual field testing, as well as baseline optical coherence tomography Ganglion Cell Analysis and 10-2 visual field testing. As a brief review, the importance (and benefits) of central 10-degree visual field analysis cannot be overstated: approximately $50 \%$ of eyes with mild-moderate glaucoma were found to have defects within the central 3 degrees, ${ }^{6} 11$ eyes with normal 24-2 visual fields outside the central 10 degrees showed arcuate defects within the central 10 degrees with 10-2 testing, ${ }^{7}$ nine percent of normal 30-2 threshold visual fields in glaucoma 
suspect or early glaucoma patients were actually classified as abnormal with 10-2 testing ${ }^{8}$ and approximately $50 \%$ of eyes will show macular glaucomatous damage on 10-2 testing while being classified as normal with just 24-2 testing. ${ }^{9}$

\section{DISCUSSION}

Weinreb et al. proposed a glaucoma continuum - a spectrum of structural and functional stages in glaucoma in which the patient generally progresses from "normal" and asymptomatic disease to functional blindness - with structural glaucomatous changes usually preceding functional symptoms. ${ }^{10}$ The World Glaucoma Association also described this temporal relationship between structural and functional changes throughout the course of the disease $\mathrm{e}^{11}$ and both representations suggest that structural changes are usually detected prior to functional changes. However, and as an important reminder from Alasil et al.'s retrospective study and their findings of a structural and functional "tipping point", both structural and functional tests are still necessary to assess early glaucomatous damage. ${ }^{12}$

The American Academy of Ophthalmology Preferred Practice Pattern (AAO PPP) for Primary Open-Angle Glaucoma states that mild (early) glaucoma is characterized by "optic nerve abnormalities consistent with glaucoma [such as] ... diffuse thinning, focal narrowing, or notching of the optic disc rim, especially at the inferior or superior poles; progressive narrowing of the neuroretinal rim with an associated increase in cupping of the optic disc; diffuse or localized abnormalities of the parapapillary RNFL [retinal nerve fiber layer], especially at the inferior or superior poles; disc rim, parapapillary RNFL, or lamina cribrosa hemorrhages; [and/or] optic disc neural rim asymmetry of the two eyes consistent with loss of neural tissue" in the presence of "a normal visual field as tested with standard automated perimetry." ${ }^{\prime 3}$

In the American Optometric Association Optometric Clinical Practice Guidelines (AOA CPG), mild glaucoma is defined as an optic nerve with "mild concentric narrowing or partial localized narrowing of the neuroretinal rim; disc hemorrhage; [and/or] cup/disc asymmetry". Furthermore, the nerve fiber layer shows a "less bright reflex; fine striations to texture; [and/or] large retinal blood vessels [that appear relatively] clear [whereas] medium retinal blood vessels [are] less blurred [and] small retinal blood vessels [are] blurred". However, unlike the AAO PPP, the AOA CPG says that early glaucoma may show "isolated paracentral scotomas, partial arcuate or nasal step [defects]; [and that the] damage [is] limited to one hemifield with fewer than $25 \%$ of points involved, [with a] mean deviation (MD) less than -6dB". ${ }^{14}$ A more succinct definition that seems to help bridge the two above definitions is given by Song and Caprioli: "a progressive optic neuropathy that is defined by characteristic structural changes of the optic nerve with corresponding functional changes of the visual field." 15

Nonetheless, once functional loss is detectable, the severity of the glaucomatous optic neuropathy increases with the severity of the visual field loss, as shown by $\mathrm{Ng}$ et al. ${ }^{16}$

The present patient presented with several risk factors for open-angle glaucoma, ${ }^{17,18}$ including his slightly elevated intraocular pressure with mild fluctuation ${ }^{19}$ (albeit based on only two isolated readings) and his mid-advanced age. ${ }^{20,21}$ However, additional risk factors that were not applicable to this case, but which should also be considered, include presence of lenticular exfoliation, ${ }^{22,23}$ glaucomatous disc hemorrhages, ${ }^{24-28}$ African-American ancestry, ${ }^{29}$ a first-degree history of glaucoma, ${ }^{30}$ and a general history of diabetes ${ }^{31}$ or hypertension..$^{32-34}$

The primary clinical sign that was most convincing that this patient did indeed have glaucoma was the appearance of the optic nerve and retinal nerve fiber layer. Specifically, the inferior retinal nerve fiber layer defects with associated inferior rim thinning, inferior vessel baring, and inferior arteriole narrowing (and potential superior rim thinning) are all characteristic of early glaucoma. Because of this preferential pattern of neuroretinal rim loss, the ISNT Rule ${ }^{35,36}$ mnemonic has been proven to be very effective in differentiating normal optic nerves from those with early glaucomatous damage. Furthermore, the absence of rim pallor helps rule out other optic neuropathies (ischemic, infiltrative, traumatic, toxic, metabolic, and compressive) that could also result in retinal nerve fiber layer defects and arteriole narrowing, and which would necessitate a more through systemic workup, possibly including blood work and neuroimaging. ${ }^{37-39}$ Baseline photos were taken to assist in monitoring for future structural progression ${ }^{40}$ that would manifest as widening of the nerve fiber layer defects (locations of future progression and correlating visual field defects ${ }^{41}$ ), increased rim thinning/ vessel baring/arteriole narrowing, increased parapapillary atrophy, and/or further nasalization of the central retinal vessel trunk. 
Subjective evaluation of serial photos and objective imaging (OCT) are complementary structural evaluations used in concert with regular functional (visual field) assessment to monitor for progression. All are necessary: the World Glaucoma Association notes that “(c)urrently, no specific test can be regarded as the perfect reference standard for detection of glaucomatous structural and/or functional progression". ${ }^{41}$ Supporting this position, Banegas et al. reported that, in their observational study of 246 eyes, glaucomatous progression was detected in $6.9 \%$ of eyes by stereo photos, $15 \%$ of eyes by visual field testing, and $25.6 \%$ of eyes by OCT-guided progression analysis (GPA) software. Interestingly, of those cases that showed progression, most were only discovered by either stereophotos, perimetry, or OCT testing alone, suggesting a lower percentage of positive agreement among evaluation methods, and emphasizing the importance of using all three to monitor for change. ${ }^{42,43}$ In this situation, the clinical appearance of the ONH correlated very well with the baseline RNFL OCT testing, establishing supporting subjective and objective structural baselines.

In support of making a diagnosis of glaucoma based solely on the appearance of the optic nerve and not waiting for the development of correlating glaucomatous visual field defects, Sommer et al. suggested in 1977 that glaucomatous nerve fiber layer defects (such as those observed in this patient) may develop several years prior to reliable glaucomatous visual field defects. ${ }^{44}$ Furthermore, and more recently, Kuang et al. found that RNFL defects observed on OCT testing were noted up to 8 years prior to associated glaucomatous visual field defects. ${ }^{45}$ Consistent with these findings, histological studies have found that as much as $50 \%$ of retinal ganglion cells are lost prior to clinically detectable visual field defects ${ }^{46}$ - resulting in a "broken-stick" correlation model between retinal nerve fiber thickness and glaucomatous visual fields, as described previously by Alasil et al. ${ }^{12}$

As mentioned previously, despite the clinically correlating information suggesting early open-angle glaucoma, collaboration with the patient determined that we did not initiate treatment for the following 3 reasons:

1. To establish a baseline IOP range in light of potential fluctuation in initial IOP measurements,

2. To establish baseline visual field reproducibility and reliability, ${ }^{47}$

3. To establish rate of progression, in recognition of the fact that not all patients with glaucoma will progress to the point of visual symptoms affecting their activities of daily living. ${ }^{48}$

\section{CONCLUSION}

Elevated intraocular pressure is the primary (and currently the only readily modifiable) risk factor for the development of glaucoma and glaucoma progression. ${ }^{49-53}$ Accordingly, if treatment is required in the future, we will work with the patient to establish a customized, unique target IOP range - the "upper limit of a range of IOP at which it is judged likely to retard further optic nerve damage" 54 and to minimize associated visual field loss. ${ }^{55}$ It is very important to balance this dynamic IOP range with quality-of-life factors including the estimated lifetime risk of visual disability for the patient, the potential side-effects of treatment options (topical vs. laser vs. minimally-invasive glaucoma surgery), the financial burden of treatment, and the instillation technique/capability.

Primary open angle glaucoma can be missed in its early stages due to its asymptomatic nature, subtle optic nerve morphological changes, and often pre-perimetric presentation. For these reasons, vigilance is required, as we have the best success of preserving a lifetime of functional vision for the patient if we can diagnose glaucoma earlier and, if needed, treat glaucoma sooner. 


\section{REFERENCES}

1. Quigley H, Broman A. The number of people with glaucoma worldwide in 2010 and 2020. Br J Ophthalmol March 2006;90(3):262-7.

2. Weinreb R, Aung T, Medeiros F. The pathophysiology and treatment of glaucoma: a review. JAMA May 14, 2014;311(18):1901-11.

3. Hennis A, Wu S, Nemesure B, Honkanen R, Leske M. Awareness of incident open-angle glaucoma in a population study: the Barbados Eye Studies. Ophthalmology October 2007;114(10):1816-21.

4. Bengtsson B, Heijl A. SITA Fast, a new rapid perimetric threshold test. Description of methods and evaluation in patients with manifest and suspect. Acta Ophthalmol Scand 1998;76:431-7.

5. Kim KO, Jeoung JW, Kim DM, et al. Long-term follow-up in preperimetric open-angle glaucoma: progression rates and associated factors. Am J Ophthalmol 2015 Jan;159(1):160-8.el-2

6. Schiefer U, Papageorgiou E, Sample PA, et al. Spatial pattern of glaucomatous visual field loss obtained with regionally condensed stimulus arrangements. Invest Ophthalmol Vis Sci 2010;51(11):5685-9.

7. Hood DC, Raza AS, de Moraes CG, et al. Initial arcuate defects within the central 10 degrees in glaucoma. Invest Ophthalmol Vis Sci 2011;52(2):940-6.

8. Langerhorst CT, Carenini LL, Bakker D, De Bie-Raakman MAC. Measurements for description of very early glaucomatous field defects. Perimetry Update 1996/1997. New York, NY: Kugler Publications;1997:67-73.

9. Grillo L, Wang D, Hood D, et al. The 24-2 visual field test misses central macular damage confirmed by the 10-2 visual field test and optical coherence tomography. Transl Vis Sci Technol $2016 \mathrm{Apr}$ $14 ; 5(2): 15$

10. Weinreb RN, Friedman DS, Fechtner RD, et al. Risk assessment in the management of patients with ocular hypertension. Am J Ophthalmol 2004 Sep;138(3):458-67.

11. Harwerth RS. Histopathology underlying glaucomatous damage - I in Glaucoma Diagnosis Structure and Function Consensus Series 1. The Hague, The Netherlands; Kugler Publications: 2004:p14.

12. Alasil T, Wang K, Yu F, Field MG. Correlation of retinal nerve fiber layer thickness and visual fields in glaucoma: a broken stick model. Am J Ophthalmol 2014;157 (5)953-9.

13. Preferred Practice Pattern Guidelines. Primary Open-Angle Glaucoma. American Academy of Ophthalmology; 2010.

14. American Optometric Association Clinical Practice Guidelines; 2010: 43 .

15. Song BJ, Caprioli J. Measuring glaucoma progression in clinical practice in Glaucoma - Medical Diagnosis and Therapy. Elsevier Limited 2015. Chapter 23:268-76.

16. Ng M, Sample PA, Pascual JP, et al. Comparison of visual field severity classification systems for glaucoma. J Glaucoma 2012; 21(8):551-61.

17. Chen PP. Risk and risk factors for blindness from glaucoma. Curr Opin Ophthalmol 2004;15(2):107-11.

18. Fechtner RD, Khouri AS. Evolving global risk assessment of ocular hypertension to glaucoma. Curr Opin Ophthalmol 2007;18(2):104-9.

19. Wilensky JT, Gieser DK, Dietsche ML, et al. Individual variability in the diurnal intraocular pressure curve. Ophthalmology 1993;100(6):940-4.

20. Leske MC, Connell AM, Wu SY, et al. Risk factors for openangle glaucoma. The Barbados Eye Study. Arch Ophthalmol 1995;113(7):918-24.

21. Leske MC, Wu SY, Hennis A, et al. Risk factors for incident open-angle glaucoma: the Barbados Eye Studies. Ophthalmology 2008;115(1):85-93.

22. Jeng SM, Karger RA, Hodge DO, Burke JP, Johnson DH, Good MS The risk of glaucoma in pseudoexfoliation syndrome. J Glaucoma 2007;16(1):117-21.

23. Grødum K, Heijl A, Bengtsson B. Risk of glaucoma in ocular hypertension with and without pseudoexfoliation. Ophthalmology 2005;112(3): 386-90

24. Suh MH, Park KH. Pathogenesis and clinical implication of optic disk hemorrhage in glaucoma. Surv Ophthalmol 2014; 59:19-29.

25. Healey P, Mitchell P, Smith W, Wang J. Optic disc hemorrhages in a population with and without signs of glaucoma. Ophthalmology February 1998;105(2):216-23.

26. Siegner SW, Netland PA. Optic disc hemorrhages and progression of glaucoma. Ophthalmology 1996;103:1014-24.

27. Uhler T, Piltz-Seymour J. Optic disc hemorrhages in glaucoma and ocular hypertension: implications and recommendations. Curr Opin Ophthalmol March 2008;19(2):89-94.

28. Suh M, Park K. Period prevalence and incidence of optic disc haemorrhage in normal tension glaucoma and primary open-angle glaucoma. Clin Exp Ophthalmol August 2011;39(6):513-9.

29. Tielsch JM, Sommer A, Katz J, et al. Racial variations in the prevalence of primary open-angle glaucoma. The Baltimore Eye Survey. JAMA 1991;266(3):369-74.

30. Gordon MO, Torri V, Miglior S, et al. Validated prediction model for the development of primary open-angle glaucoma in individuals with ocular hypertension. Ophthalmology 2007;114(1):10-19.

31. Zhou M, Wang W, Huang W, Zhang X. Diabetes mellitus as a risk factor for open-angle glaucoma: a systematic review and metaanalysis. PLoS One August 19,2014;9(8):e102972.

32. Tielsch JM, Katz J, Sommer A, et al. Hypertension, perfusion pressure, and primary open-angle glaucoma. A population-based assessment. Arch Ophthalmol 1995;113(2):216-21.

33. Fechtner RD, Khouri AS. Evolving global risk assessment of ocular hypertension to glaucoma. Curr Opin Ophthalmol 2007;18(2):104-9.

34. Zhao D, Cho J, Kim MH, Guallar E. The association of blood pressure and primary open-angle glaucoma: a meta-analysis. Am J Ophthalmol 2014;158:615-27.

35. Jonas J, Gusek G, Naumann G. Optic disc, cup and neuroretinal rim size, configuration and correlations in normal eyes. Invest Ophthalmol Vis Sci July 1988;29(7):1151-8.

36. Harizman N, Oliveira C, Liebmann J, et al. The ISNT rule and differentiation of normal from glaucomatous eyes. Arch Ophthalmol (Chicago, Ill.: 1960). November 2006;124(11):1579-83.

37. Choudhari N, Neog A, Fudnawala V, George R. Cupped disc with normal intraocular pressure: the long road to avoid misdiagnosis. Indian J Ophthalmol November 2011;59(6):491-7.

38. Greenfield D, Siatkowski R, Glaser J, Schatz N, Parrish R. The cupped disc. Who needs neuroimaging? Ophthalmology October 1998;105(10):1866-74.

39. Greenfield D. Glaucomatous versus nonglaucomatous optic disc cupping: clinical differentiation. Semin Ophthalmol June 1999;14(2):95-108.

40. Zeyen T, Miglior S, Pfeiffer N, et al. Reproducibility of evaluation of optic disc change for glaucoma with stereo optic disc photographs. Ophthalmology 2003; 110(2):340-4.

41. Weinreb R, World Glaucoma A. Progression of Glaucoma: The $8^{\text {th }}$ Consensus Report of the World Glaucoma Association. Amsterdam: Kugler Publications; 2011, 91-9.

42. Banegas S, Antón A, Morilla-Grasa A, Bogado M, Ayala E, MorenoMontañes J. Agreement among spectral-domain optical coherence tomography, standard automated perimetry, and stereo photography in the detection of glaucoma progression. Invest Ophthalmol Vis Sci January 27, 2015;56(2):1253-60.

43. Sehi M, Bhardwaj N, Chung YS, Greenfield DS; Advanced Imaging for Glaucoma Study Group. Evaluation of baseline structural factors for predicting glaucomatous visual-field progression using optical coherence tomography, scanning laser polarimetry and confocal scanning laser ophthalmoscopy. Eye (Lond). 2012 Dec;26(12):1527-3. 